\title{
LkCa 15: A YOUNG EXOPLANET CAUGHT AT FORMATION?
}

\author{
Adam L. Kraus ${ }^{1,6}$ and Michael J. Ireland ${ }^{2,3,4,5}$ \\ ${ }^{1}$ Institute for Astronomy, University of Hawaii at Manoa, 2680 Woodlawn Dr., Honolulu, HI 96816, USA \\ 2 Macquarie University Research Centre in Astronomy, Astrophysics, \& Astrophotonics, NSW 2109, Australia \\ ${ }^{3}$ Department of Physics and Astronomy, Macquarie University, NSW 2109, Australia \\ ${ }^{4}$ Australian Astronomical Observatory, P.O. Box 296, Epping, NSW 1710, Australia \\ 5 Sydney Institute for Astronomy (SIfA), School of Physics, NSW 2006, Australia \\ Received 2011 August 1; accepted 2011 October 2; published 2011 December 27
}

\begin{abstract}
Young and directly imaged exoplanets offer critical tests of planet-formation models that are not matched by radial velocity surveys of mature stars. These targets have been extremely elusive to date, with no exoplanets younger than 10-20 Myr and only a handful of direct-imaged exoplanets at all ages. We report the direct-imaging discovery of a likely (proto)planet around the young $(\sim 2 \mathrm{Myr})$ solar analog LkCa 15 , located inside a known gap in the protoplanetary disk (a "transitional disk"). Our observations use non-redundant aperture masking interferometry at three epochs to reveal a faint and relatively blue point source $\left(M_{K^{\prime}}=9.1 \pm 0.2, K^{\prime}-L^{\prime}=0.98 \pm 0.22\right)$, flanked by approximately co-orbital emission that is red and resolved into at least two sources $\left(M_{L^{\prime}}=7.5 \pm 0.2\right.$, $K^{\prime}-L^{\prime}=2.7 \pm 0.3 ; M_{L^{\prime}}=7.4 \pm 0.2, K^{\prime}-L^{\prime}=1.94 \pm 0.16$ ). We propose that the most likely geometry consists of a newly formed (proto)planet that is surrounded by dusty material. The nominal estimated mass is $\sim 6 M_{\text {Jup }}$ according to the 1 Myr hot-start models. However, we argue based on its luminosity, color, and the presence of circumplanetary material that the planet has likely been caught at its epoch of assembly, and hence this mass is an upper limit due to its extreme youth and flux contributed by accretion. The projected separations $(71.9 \pm 1.6$ mas, $100.7 \pm 1.9$ mas, and $88.2 \pm 1.8$ mas) and deprojected orbital radii $(16,21$, and $19 \mathrm{AU})$ correspond to the center of the disk gap, but are too close to the primary star for a circular orbit to account for the observed inner edge of the outer disk, so an alternative explanation (i.e., additional planets or an eccentric orbit) is likely required. This discovery is the first direct evidence that at least some transitional disks do indeed host newly formed (or forming) exoplanetary systems, and the observed properties provide crucial insight into the gas giant formation process.
\end{abstract}

Key words: planets and satellites: detection - planets and satellites: formation - protoplanetary disks - stars: individual: LkCa 15 - stars: pre-main sequence

Online-only material: color figures

\section{INTRODUCTION}

In the past 15 years, indirect searches for extrasolar planets (i.e., radial velocity and transit surveys) have discovered over 500 confirmed planetary companions to other stars (Wright et al. 2011), spurring explosive growth in the field of comparative exoplanetology. However, virtually all of these planets orbit around old stars ( $\tau \geqslant 1 \mathrm{Gyr})$, typically at orbital radii smaller than that of Jupiter. Direct detection via high-resolution imaging holds great promise for extending comparative exoplanetology across the full range of planetary ages and orbital radii. Direct detection programs are vital for studying the outer regions of extrasolar systems (where analogs to our own gas giant planets should reside) since those planets are inaccessible to transit searches and can only be discovered with decadeslong surveys by radial velocity programs. Direct detection also offers a window into the detailed atmospheric and evolutionary properties of exoplanets since they are amenable to photometric and spectroscopic study. Finally, young exoplanets should be much hotter and more luminous than their older counterparts (Marley et al. 2007; Fortney et al. 2008), so they are easier to detect and should offer a window into the very early stages of planet formation and evolution. Direct-imaging techniques still face significant technical challenges, but those challenges are being overcome with innovative new observational techniques

\footnotetext{
6 Hubble Fellow.
}

that have begun to yield the first discoveries (Marois et al. 2008; Kalas et al. 2008; Lagrange et al. 2009).

Young planets and outer planets will provide critical new tests of the formation and evolution of planetary systems, as the two competing models of planet formation ("core accretion," Pollack et al. 1996, and "disk instability," Boss 2001) make very different claims about when and where planets should form. Disk instability should preferentially form planets in outer solar systems, and typically will do so within $\sim 1 \mathrm{Myr}$ after a star has formed. In contrast, core accretion is much more efficient in forming planets with small orbital radii, and the assembly process should require 3-5 Myr to form gas giants analogous to our own Jupiter and Saturn. Furthermore, almost all gas giant planets are expected to migrate both inward and outward in their orbits due to gravitational interactions with the protoplanetary disk and with smaller rocky bodies (Ida \& Lin 2004; Tsiganis et al. 2005). Almost all of the gas giant planets around solar-type stars with orbital radii of $\leqslant 2-3$ AU are thought to have formed at larger radii and migrated inward, so their frequency and orbital properties are not necessarily representative of most systems. The most demanding tests of planet formation models can come only from direct observations of young planetary systems as they form.

We have learned much about planet formation from other advances over the past decade. Mid- and far-infrared observations with the Spitzer Space Telescope have cast new light on the formation, evolution, and lifetimes of the circumstellar 
disks where planets form (Hillenbrand 2008). Similarly, new observations by millimeter/submillimeter observatories have established the masses and sizes of disks, especially on spatial scales of 10-100 AU where dust is too cool to emit in the mid-IR or far-IR (Andrews \& Williams 2005; Andrews et al. 2011). In both cases, observations have also discovered a rare, intriguing class of objects: protoplanetary disks where the mid-IR spectral energy distributions (SEDs) or resolved images (from submillimeter/millimeter wavelengths) reveal gaps or inner holes (Calvet et al. 2005; Espaillat et al. 2007; Brown et al. 2009). These gaps could be cleared by the gravitational influence of other bodies (Ireland \& Kraus 2008; Huelamo et al. 2011) such as stellar binaries or gas giant planets. In cases where binary companions are ruled out, then these gaps serve as signposts of likely ongoing planet formation. These likely sites of planet formation are natural targets for pushing the boundaries of observational capabilities. Giant planets should be present, and the geometry of the disk gaps can even demonstrate their locations. To this end, we have begun a survey to directly image exoplanets inside the gaps of protoplanetary disks using a recently developed technique, non-redundant mask interferometry (NRM).

One of the first targets we chose for our survey was the young $\left(2_{-1}^{+2}\right.$ Myr; Kraus \& Hillenbrand 2009) solar analog LkCa 15, which is located in the Taurus-Auriga star-forming region and is known to have a massive $\left(55 M_{\text {Jup }}\right.$ ) circumstellar disk (Andrews \& Williams 2005). Detailed modeling of the disk's mid-infrared spectrum has demonstrated that it appears to have a sizeable gap (Espaillat et al. 2007). LkCa 15 shows near-infrared emission from warm dust in the inner $<1 \mathrm{AU}$, plus mid- and far-infrared emission from cold dust outside $>50$ AU. However, there is a deficit of emission at 10-20 $\mu \mathrm{m}$, indicating the presence of a gap at intermediate radii that is cleared of dust. Subsequent observations at longer wavelengths that directly trace the dust also demonstrate a deep paucity of emission at separations of $<55$ AU (Andrews et al. 2011), and near-infrared imaging may have observed reflected light from the inner edge of the disk (Thalmann et al. 2010). We previously observed LkCa 15 with NRM in the $K^{\prime}$ band to determine if this cleared region could indicate the presence of a binary companion (Kraus et al. 2011), but found no companions with a mass of $>12 M_{\text {Jup }}$. We therefore made the system a high priority for our planet-search program, which is obtaining significantly longer observations than our previous study ( $\geqslant 4 \mathrm{hr}$ versus $\sim 20$ minutes).

In this paper, we report the discovery of a likely (proto)planet orbiting LkCa 15, which we have caught at the epoch of formation. In Section 2, we describe our observations of LkCa 15 and the methods we used to analyze our data. In Section 3, we describe our discovery of the apparent (proto)planetary companion and circumplanetary material, its apparent properties, and the alternative explanations for our observations that we are able to rule out. Finally, in Section 4, we discuss some of the implications of our discovery for planet formation and for the nature of transitional disks.

\section{OBSERVATIONS AND DATA ANALYSIS}

\subsection{Non-redundant Mask Interferometry of LkCa 15}

The sensitivity of adaptive optics imaging for close companions $(<2-3 \lambda / D)$ is limited by imperfect calibration of the primary star's point-spread function (PSF); the PSF width and shape change under different atmospheric conditions, and quasistatic image artifacts ("speckles," which resemble faint com- panions) are superimposed on the image by uncorrected atmospheric turbulence and by optical imperfections in the telescope itself. The technique of NRM has been well established as a means of achieving the full diffraction limit of a single telescope (Nakajima et al. 1989; Tuthill et al. 2000, 2006). NRM uses a pupil-plane mask to block most of the light from a target, resampling the primary mirror into a set of smaller subapertures that form a sparse interferometric array. Rather than an image of the target, the science camera then observes its interferometric fringes. NRM allows for superior calibration of the stellar primary's point spread function and elimination of speckle noise by the application of interferometric analysis techniques. In particular, the measurement of closure phases allows for strehlindependent calibration of the stellar PSF and the canceling of low-order phase errors that cause speckle noise in conventional imaging. NRM observations can yield contrasts of $\Delta K \sim 6 \mathrm{mag}$ at $\lambda / D$ and $\Delta K \sim 4$ mag at $1 / 3 \lambda / D$ even in very short observations. Some of the unique results of past high-contrast NRM observations include a measurement of one of the first dynamical masses for a brown dwarf, GJ 802 B (Ireland et al. 2008), several studies of the multiplicity of young stars (Ireland et al. 2008; Ireland \& Kraus 2008), and the discovery of a potentially substellar candidate companion to another transitional disk host, T Cha (Huelamo et al. 2011).

We observed LkCa 15 over the course of three observing runs using the Keck-II $10 \mathrm{~m}$ telescope in 2009 November, 2010 August, and 2010 November. All observations were conducted with the facility AO imager, NIRC2, which has aperture masks installed in the cold filter wheel near the pupil stop. All of our observations used a nine-hole aperture mask, which passes $11 \%$ of the total incident flux through nine $1.1 \mathrm{~m}$ subapertures that span baselines of 1.5-9.2 $\mathrm{m}$. This choice maximizes the throughput as the other option (an 18 hole mask) passes half as much incident flux and can only be used with narrowband filters (due to wavelength-dependent dispersion in broadband filters) that are $\sim 10 \%$ as wide as the corresponding broadband filters. The nine subapertures yield 28 independent baseline triangles about which closure phases are measured. All NRM observations operate in a subarray mode of the narrow camera, which has a pixel scale of $9.963 \mathrm{~ms} \mathrm{pix}^{-1}$, and we conducted our observations using the $L^{\prime}(3.43-4.13 \mu \mathrm{m})$ and $K^{\prime}(1.96-2.29 \mu \mathrm{m})$ broadband filters. The observations spanned most of several nights, so LkCa 15 was observed at airmasses ranging from 1.0 to 1.9 . We summarize the average seeing on each night in Table 1.

Each observing sequence consisted of multiple "visits" of LkCa 15, alternating with observations of independent calibrator stars. These calibrators are essential PSF calibrators, which for aperture-masking observations are used to estimate the systematic non-zero closure-phases (e.g., third-order effects of phase aberrations within each subaperture). We chose these calibrators to be near LkCa 15 on the sky ( $<7^{\circ}$ separation) and to have similar brightness in the optical (for similar adaptive optics performance) and to be brighter in the near-infrared (so that the Poisson noise would be small compared to that from $\mathrm{LkCa} 15)$. The number of observations taken at each epoch and the calibrators used are given in Table 1 . All of these stars were chosen from our previous binary survey (Kraus et al. 2011) and are known to have no stellar companions at angular separations of $>20$ mas and no brown dwarf companions at $>40$ mas. Each individual calibrator is observed much less often than LkCa 15, so any calibration error from faint companions to the calibrators should be negligible. 
Table 1

Observing Log for $\mathrm{LkCa} 15$

\begin{tabular}{|c|c|c|c|c|}
\hline Epoch (UT Date) & Filter & Visits $^{\mathrm{a}}$ & Seeing $^{b}$ & Calibrators $^{\mathrm{c}}$ \\
\hline 2009 Nov 19 & $L^{\prime}$ & 6 & 0.9 & GM Aur (x10), HD 283572, HP Tau/G2 (x3), UX Tau (x6), V819 Tau \\
\hline 2009 Nov 20 & $L^{\prime}$ & 7 & 0.5 & DG Tau (x2), DR Tau (x2), HP Tau/G2 (x4), UX Tau (x8) \\
\hline 2010 Aug 16 & $L^{\prime}$ & 3 & 0.5 & GK Tau, HP Tau/G2 \\
\hline 2010 Aug 17 & $L^{\prime}$ & 2 & 0.5 & DO Tau, HP Tau/G2 \\
\hline 2010 Nov 26 & $K^{\prime}$ & 12 & 0.6 & CI Tau (x2), DO Tau (x2), DQ Tau, DS Tau, GK Tau (x2), HP Tau/G2 (x3), UX Tau (x2) \\
\hline 2010 Nov 27 & $L^{\prime}$ & 4 & 0.8 & DO Tau, DS Tau, GK Tau, HP Tau/G2, SU Aur, UX Tau \\
\hline
\end{tabular}

Notes.

a Each "visit" is a single observation consisting of 20 images (for $L^{\prime}$ ) or 12 images (for $K^{\prime}$ ), each of which has an integration time of $20 \mathrm{~s}$.

$\mathrm{b}$ The seeing measurement we report is average value measured by the Canada-France-Hawaii Telescope seeing monitor during the time of the observations, and is measured in the $V$ filter.

c When calibrators were observed multiple times in one night, we denote this with a number in parentheses beside that calibrator's name.

Each "visit" consisted of a long sequence of exposures. In the $L^{\prime}$ observations, we obtained 20 exposures of $20 \mathrm{~s}$ each, yielding a total integration time of $400 \mathrm{~s}$. Data were taken in a two-point dither mode with the interferogram in opposite quadrants of the $512 \times 512$ subarray of the NIRC 2 camera. We used this subarray mode to minimize readout overhead. In the $K^{\prime}$ observations, we obtained 12 exposures of $20 \mathrm{~s}$ per visit because we expected calibration error to be more significant at $K^{\prime}$ than at $L^{\prime}$ (due to the lower strehls delivered by the AO system), and thus we wanted the science and calibration observations to be as simultaneous as possible. We also did not dither, since the thermal backgrounds at $K^{\prime}$ are negligible given the brightness of our targets (unlike for $L^{\prime}$ ) and therefore sky subtraction was unnecessary. We summarize the history of all "visits" in Table 1.

\subsection{Data Analysis}

The data analysis up to the calibration step was identical to that used in previous papers (Ireland et al. 2008; Kraus et al. 2008). However, most of the subsequent steps were developed or refined when we discovered that our initial fits (for a single point-source companion) had high residuals, indicating the possibility of more complex structure. To briefly summarize the identical initial steps, the images were flat-fielded and bad pixels were removed by interpolating between neighboring pixels. The image was then multiplied by a super-Gaussian window function of the form $\exp \left(-a r^{4}\right)$, with $r$ the radius in pixels from the center of mass of the interferogram. A two-dimensional Fourier transform was then made of each exposure in a visit, and this Fourier transform was point-sampled at the positions corresponding to the baseline vectors in the aperture mask. For visit $k$ we computed the vector of mean uncalibrated closurephases $\phi_{k}$ and the standard error of the mean $\sigma_{k}\left(\phi_{k}\right)$.

In the past, we have generally calibrated the closure phase simply by subtracting off the uncalibrated closure-phases measured for calibrator stars observed closest in time. We initially used this method for analysis in this paper, detecting the same key structures as reported below. An independent analysis of the 2009 epoch using the SAMP code (Lacour et al. 2011) also produced consistent results (S. Lacour 2011, private communication). However, deciding on which calibrators are "closest" in time and how many to use is a somewhat arbitrary process, and in addition, we must consider variable atmospheric dispersion in the $L$-band (as discussed in Hinkley et al. 2011).

For results reported here, we improved our previously used technique by choosing an optimal linear combination of calibrators for each target star visit. The detailed motivation for this algorithm will be described in M. J. Ireland (in prepara- tion), but we describe the essential components of the algorithm here. First, rather than using correlated closure-phases as the primary observable in the fitting algorithm, we used statistically independent linear combinations of closure-phases $\boldsymbol{x}_{\boldsymbol{k}}$. This is a similar approach to that used by Martinache (2010) for his kernel phases, however, we guarantee statistical independence using the measured closure-phase covariance matrix rather than assuming all Fourier phases to be independent. Our optimal linear combination of calibrators is such that the sum of the mean-square residuals of calibrated observables $\boldsymbol{x}_{\boldsymbol{t}}-\sum_{k=1}^{N_{c}} a_{k} \boldsymbol{x}_{\boldsymbol{k}}$ and the estimated variance in our observables is minimized. The sum is given by

$$
S=\left|\frac{\boldsymbol{x}_{\boldsymbol{t}}-\sum_{k=1}^{N_{c}} a_{k} \boldsymbol{x}_{\boldsymbol{k}}}{\boldsymbol{\sigma}^{\mathbf{2}}\left(\boldsymbol{x}_{\boldsymbol{t}}\right)+\Delta_{t}^{2}}\right|^{2}+\left|\frac{\sum_{k=1}^{N_{c}} a_{k}^{2}\left(\boldsymbol{\sigma}_{\boldsymbol{k}}^{2}\left(\boldsymbol{x}_{\boldsymbol{k}}\right)+\Delta_{t}^{2}\right)}{\boldsymbol{\sigma}^{\mathbf{2}}\left(\boldsymbol{x}_{\boldsymbol{t}}\right)+\Delta_{t}^{2}}\right|^{2} .
$$

Here, $N_{c}$ is the number of calibrators observed on a night, $\boldsymbol{x}_{\boldsymbol{t}}$ is the vector of target closure-phase combinations being calibrated, the calibrator weights are $a_{k}$ and the systematic error component is $\Delta_{t}$. The systematic error component $\Delta_{t}$ is set so that the reduced chi-squared for each target visit is no more than unity. Like the LOCI algorithm used to reduce AO direct-imaging data (Lafrenière et al. 2007), finding an optimal linear combination of calibrators in this way tends to reduce the significance of a real companion in the data. For this reason, for the fits consisting of several point sources we used this technique to determine if any given model was significant, then re-computed the calibration optimization using Equation (1) where the target phase combinations $\boldsymbol{x}_{\boldsymbol{t}}$ had the phases of the best-fit global model subtracted (with three additional companions: see below). The difference this updated calibration caused to the final fitted parameters was less than $1.5 \sigma$ in all cases.

Typical values of $\Delta_{t}$ required to achieve unity reduced chisquared were comparable to or larger than the $\sim 0.8$ deg closurephase scatter in the $L^{\prime}$ filter when the target phase combinations $\boldsymbol{x}_{\boldsymbol{t}}$ did not include the best-fit model, but were zero $\sim 40 \%$ of the time and always smaller than the internal scatter when the target phase combinations $\boldsymbol{x}_{\boldsymbol{t}}$ had the best-fit model subtracted. Comparison stars (i.e., calibrating the calibrators from Table 1) also had small or zero values for $\Delta_{t}$ and calibrated closure-phase uncertainties of order $0.8 \mathrm{deg}$ for the $L^{\prime}$ filter and $0.4 \mathrm{deg}$ for the $K^{\prime}$ filter in each visit. These uncertainties are much lower than the uncalibrated closure-phases, which had typical median absolute values of $\sim 4 \mathrm{deg}$ for the $L^{\prime}$ filter and $\sim 2 \mathrm{deg}$ for the $K^{\prime}$ filter. This highlights the need for a robust closure-phase calibration process. 


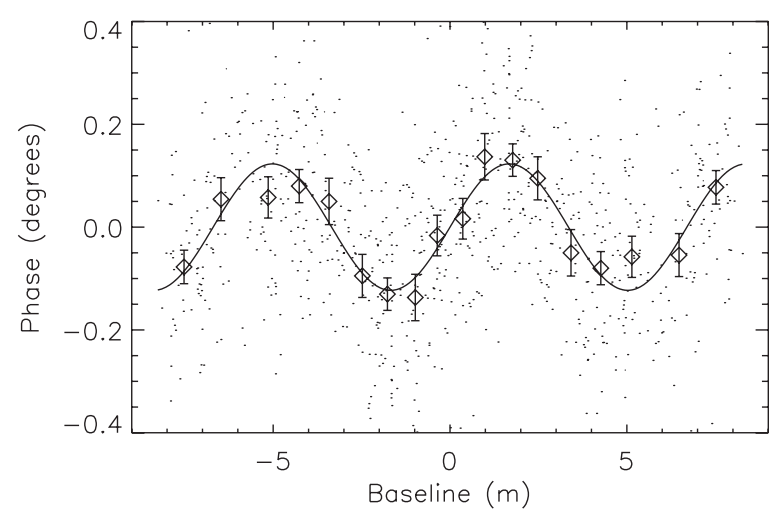

Figure 1. Fourier phase fitted to closure-phase (small dots) and the binned version of the same observable (triangles) for all $2010 \mathrm{~K}$-band data on LkCa 15 , plotted against the baseline projected along the principle axis of the best-fit binary model. The phases of the best-fit binary model from Table 2 are shown as a solid line.

It is difficult to directly show the quality of fits by directly plotting measured and model closure-phases. However, in the case of an image which is largely one-dimensional, we can fit Fourier phase to closure-phase, where the phases are chosen by a least-squares minimization process that both fits the closure-phase and attempts to fit the binary model (essentially filling in the missing phase information with the model). This is the approach of Figure 6 of Lacour et al. (2011). The phases can then be plotted along baselines projected along the principle axis of the model image. We are able to do this for the $K$-band 2010 data, which has one dominant point source in our fitting, and show this in Figure 1.

Our closure phase image reconstructions used the MonteCarlo MArkov Chain IMager algorithm (MACIM; Ireland et al. 2006). When using the mean image output, this algorithm is essentially identical to a maximum entropy method and has been used many times in optical interferometry imaging (Monnier et al. 2007; Zhao et al. 2008). The MACIM image model consisted of a point source with variable flux (i.e., free to be chosen by the algorithm) and an extended image. This point source represents the star, and without it as an explicit parameter in the model, maximum entropy-like methods spread the central source flux throughout the image, especially when visibility amplitudes are poorly constrained. For our data, visibility errors are always at least a factor of $\sim 2$ larger than closure-phase errors, which were typically less than 0.02 radians. The visibility errors were also highly correlated and vary with the adaptive optics strehl ratio. Therefore, we chose to essentially fit only to the closure-phase data, and accomplished this by adding squaredvisibility errors of 0.2 to the calibrated data. This in turn meant that we were insensitive to any point-symmetric extended flux in the image. We chose the field of view (FOV) of the images to match the window function size in our data analysis, and chose the number of image elements so that the (super-)resolution of the final image was approximately $\lambda / 2 D$.

In addition to producing reconstructed images, we also directly fit the closure phases with models described by a small number of point sources. This new multi-source fitting routine was motivated by high residuals seen in a fit with one companion (our standard technique), as well as by potentially complex structures seen in the reconstructed images. We first attempted to fit a single companion model directly to the closure phases by using a grid search, as has been standard for our previous observations. We then searched for solutions with up to three additional point-source solutions in the vicinity of the best- fit single-companion models. We used a gradient-descent least squares algorithm in up to 12 dimensions (three contrasts each in $K^{\prime}$ and/or $L^{\prime}$, three separations, and three position angles) for this fitting, making use of the IDL mpfit library. As described above, we fit to statistically independent linear combinations of closure phases and have $\chi^{2} \sim 1$ by construction (due to the $\Delta_{t}$ parameter), so we report the formal errors directly output by the mpfit program. The source(s) described here are located at $>\lambda / D$, so they are not subject to the degeneracy between contrast and separation that was seen for the similar detection of T Cha (Huelamo et al. 2011).

We also attempted to reconstruct images based on the full complex visibilities (i.e., using both amplitude and phase) in order to retrieve information on the point-symmetric extended flux (or lack thereof). The $2009 L^{\prime}$ data and $2010 K^{\prime}$ data had amplitudes too noisy to be useful in this high-contrast regime, but the $2010 L^{\prime}$ data gave images consistent with the closure-phase images that we found. We also used pointsource fits (as described above) in order to more quantitatively measure whether most flux comes from a flux-symmetric component (such as a disk) or from the asymmetric component (a companion).

The calibrated oifits files (Pauls et al. 2005) and the code that produced the fits and images for this paper have been made available at http://www.physics.mq.edu.au/ mireland/LkCa15_sup/.

\section{RESULTS}

\subsection{A (Proto)planetary Companion to LkCa 15 ?}

Our $L^{\prime}$ observations in 2009 November found a closure phase signal that was inconsistent with that of a single point source - that is, a star with no companions - at a confidence level of $>10 \sigma$. We initially fit this source with a single faint companion, and found that it significantly improved the fit in both the discovery epoch and a follow-up $L^{\prime}$ epoch in 2010 August. However, the residuals in the fit remained consistently higher for both epochs than was seen for the calibrators, hinting that the "companion" could represent a more complex structure than a single point source. A third $L^{\prime}$ observation in 2010 November confirmed that the system indeed required multiple point sources to fully explain its closure phases and visibilities. However, a deep observation in $K^{\prime}$ further complicated the picture, as those visibilities suggested most of the flux was located at a single position in between two peaks of $L^{\prime}$ flux.

In Figure 2, we show the results of independent image reconstructions of the closure phases from the 2009 November $L^{\prime}, 2010$ August $L^{\prime}, 2010$ November $L^{\prime}$, and 2010 November $K^{\prime}$ epochs. These data portray a system with complex colordependent morphology. The $L^{\prime}$ flux is seemingly dominated by two bright peaks at similar projected separations and with position angles $\sim 50$ deg apart, while most of the $K^{\prime}$ flux comes from a single point source located between the $L^{\prime}$ sources. There is some evidence for emission in $L^{\prime}$ between the two main peaks, though with less significance. The shorter $L^{\prime}$ observations (from the 2010 epochs) show more blending of the $L^{\prime}$ emission into a single elongated structure, which could indicate that these observations lack sufficient signal-to-noise ratio $(\mathrm{S} / \mathrm{N})$ to support full image reconstructions. There is also a $K^{\prime}$ peak near the position of the southwestern $L^{\prime}$ peak, suggesting a possible counterpart for that component as well.

In Table 2, we list the corresponding astrometry and photometry derived from directly fitting the closure phases of each epoch (and various combinations of them) with models 

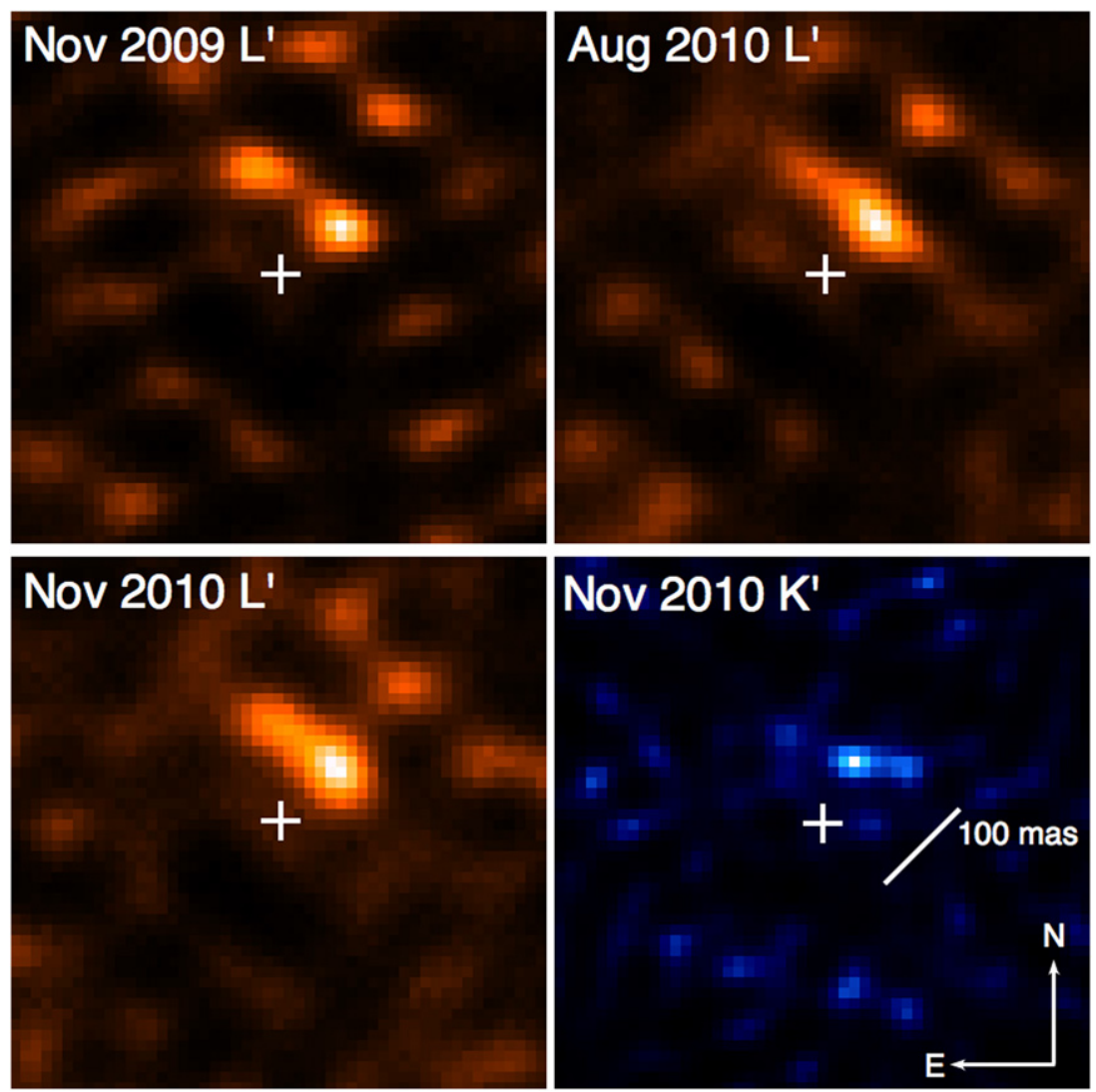

Figure 2. Reconstructed images for the four observations of LkCa 15: 2009 November $L^{\prime}$ (upper left), 2010 August $L^{\prime}$ (upper right), $2010 \mathrm{November} L^{\prime}$ (lower left), and 2010 November $K^{\prime}$ (lower right). Each image was reconstructed with a pixel scale of 10 mas (total FOV = 0'.5), and the stretch was chosen to reveal the noise peaks (most of which are aliased power from the genuine detections) without saturating the statistically significant detections (see the text). We denote the location of the central star with a cross and show a 100 mas bar for scale; all significant structures lie at or inside this angular distance from the central star. The shorter observations in $L^{\prime}$ (from 2010 August and 2010 November) show more smearing of the spatially resolved structure, indicating that the data quality is not quite sufficient for image reconstruction to be effective. However, direct fits to the closure phases (Table 2) show that the fits with a set number of point sources are typically consistent to within the uncertainties.

(A color version of this figure is available in the online journal.)

including $0,1,2$, or 3 additional point sources. This approach offers a more quantitative and sensitive measure of the system's properties, and since the entire structure is only $\sim 3-4$ times the size of the effective resolution, then a decomposition into point sources should encapsulate most of the useful morphological information. A mismatch between our model and the true complexity of the observed structure could result in systematic uncertainties, though, so the results should be treated with caution.

Each of the individual $L^{\prime}$ epochs allows for two point sources at a statistically significant level $(\sigma<0.2 \mathrm{mag}$ or $\mathrm{S} / \mathrm{N}>5)$, while combining at least two of the $L^{\prime}$ epochs allows for the fitting of three statistically significant point sources. The $K^{\prime}$ epoch also allows for a fit with three statistically significant point sources, albeit with the third source only barely significant. Given the overall morphology observed in the reconstructed images, we have classified each source as corresponding to a northeast (NE), central (CEN), or southwest (SW) component. Wavelength-dependent changes in the fit position (as for the projected separations of CEN in $L^{\prime}$ and $K^{\prime}$ ) suggest that the underlying morphology is indeed more complex, but further decomposition is not warranted by the resolution of our data. Even at this scale, it is possible that there is degeneracy between the fluxes of the flanking components and the central component, with flux from NE and SW contaminating the measurement for CEN. However, the positions of each component can be attributed to flux seen in the reconstructed images of Figure 2, suggesting that the overall morphology is being captured by both methods.

The $L^{\prime}$ visibility amplitudes are too noisy to contribute to a full image reconstruction, and even point-source fits are too noisy for the same level of detail as fits based on purely closure phases. However, in a fit to the $2010 L^{\prime}$ data with point-symmetric and antisymmetric models of three point sources (i.e., two companions), we find that the total flux is $1.7 \pm 0.3 \%$ using full visibilities and $1.4 \pm 0.1 \%$ using only the closure phases. This fit indicates that $91 \pm 9 \%$ of the total flux comes from the antisymmetric structures seen in the reconstructed images, with no more than the remaining $9 \%$ coming from a point-symmetric component (such as a disk). This strong limit shows that our observed sources do indeed represent localized structures, rather than bright clumps embedded in a disk.

Given the unusual nature of this source, we must consider the validity of the detection. The use of inappropriate (i.e., binary) calibrators can lead to the detection of spurious sources. However, we can reject this hypothesis since the companion was detected on six different nights over two years, typically using different sets of calibrators. We also tested the observations by omitting each calibrator in turn to see if the detection remained. The detection lost significance due to the smaller number of calibrators remaining, but it remained at all epochs. This same degree of persistence also allows us to reject the possibility that we are seeing a background source (which given its spatially 
Table 2

Photometry and Astrometry

\begin{tabular}{|c|c|c|c|c|c|c|c|c|c|c|c|c|}
\hline Epoch & Filter & $\begin{array}{c}\text { Sep } \\
\text { (mas) }\end{array}$ & $\begin{array}{l}\text { P.A. } \\
\text { (deg) } \\
(\mathrm{NE})\end{array}$ & $\begin{array}{c}\Delta m \\
(\mathrm{mag})\end{array}$ & $\begin{array}{c}\text { Sep } \\
\text { (mas) }\end{array}$ & $\begin{array}{c}\text { P.A. } \\
\text { (deg) } \\
(\mathrm{CEN})\end{array}$ & $\begin{array}{c}\Delta m \\
(\mathrm{mag})\end{array}$ & $\begin{array}{c}\text { Sep } \\
\text { (mas) }\end{array}$ & $\begin{array}{l}\text { P.A. } \\
\text { (deg) } \\
\text { (SW) }\end{array}$ & $\begin{array}{c}\Delta m \\
(\mathrm{mag})\end{array}$ & $\chi^{2}$ & $\overline{N_{\mathrm{df}}}$ \\
\hline \multicolumn{13}{|c|}{ No companions } \\
\hline 2009 Nov & $L^{\prime}$ & $\ldots$ & $\ldots$ & $\ldots$ & $\ldots$ & $\ldots$ & $\ldots$ & $\ldots$ & $\ldots$ & $\ldots$ & 735 & 336 \\
\hline $2010 \mathrm{Aug}$ & $L^{\prime}$ & $\ldots$ & $\ldots$ & $\ldots$ & $\ldots$ & $\ldots$ & $\ldots$ & $\ldots$ & $\ldots$ & $\ldots$ & 257 & 140 \\
\hline 2010 Nov & $L^{\prime}$ & $\ldots$ & $\ldots$ & $\ldots$ & $\ldots$ & $\ldots$ & $\ldots$ & $\ldots$ & $\ldots$ & $\ldots$ & 269 & 112 \\
\hline 2010 & $L^{\prime}$ & $\ldots$ & $\ldots$ & $\ldots$ & $\ldots$ & $\ldots$ & $\ldots$ & $\ldots$ & $\ldots$ & $\ldots$ & 525 & 252 \\
\hline $2009+2010$ & $L^{\prime}$ & $\ldots$ & $\ldots$ & $\ldots$ & $\ldots$ & $\ldots$ & $\ldots$ & $\ldots$ & $\ldots$ & $\ldots$ & 1261 & 588 \\
\hline 2010 Nov & $K^{\prime}$ & $\ldots$ & $\ldots$ & $\ldots$ & $\ldots$ & $\ldots$ & $\ldots$ & $\ldots$ & $\ldots$ & $\ldots$ & 364 & 333 \\
\hline \multicolumn{13}{|c|}{ One companion } \\
\hline 2009 Nov & $L^{\prime}$ & $\ldots$ & $\ldots$ & $\ldots$ & $77.3 \pm 3.0$ & $318.1 \pm 1.6$ & $5.61 \pm 0.07$ & $\ldots$ & $\ldots$ & $\ldots$ & 502 & 333 \\
\hline $2010 \mathrm{Aug}$ & $L^{\prime}$ & $\ldots$ & $\ldots$ & $\ldots$ & $78.3 \pm 4.4$ & $321.2 \pm 2.7$ & $5.34 \pm 0.12$ & $\ldots$ & $\ldots$ & $\ldots$ & 169 & 137 \\
\hline 2010 Nov & $L^{\prime}$ & $\ldots$ & $\ldots$ & $\ldots$ & $80.9 \pm 3.6$ & $320.6 \pm 2.0$ & $5.17 \pm 0.11$ & $\ldots$ & $\ldots$ & $\ldots$ & 154 & 109 \\
\hline 2010 & $L^{\prime}$ & $\ldots$ & $\ldots$ & $\ldots$ & $79.4 \pm 2.9$ & $320.9 \pm 1.7$ & $5.25 \pm 0.08$ & $\ldots$ & $\ldots$ & $\cdots$ & 325 & 249 \\
\hline $2009+2010$ & $L^{\prime}$ & $\ldots$ & $\ldots$ & $\ldots$ & $78.0 \pm 2.1$ & $318.7 \pm 1.2$ & $5.49 \pm 0.05$ & $\ldots$ & $\ldots$ & $\ldots$ & 837 & 585 \\
\hline 2010 Nov & $K^{\prime}$ & $\ldots$ & $\ldots$ & $\ldots$ & $65.6 \pm 1.5$ & $332.1 \pm 1.2$ & $6.67 \pm 0.09$ & $\ldots$ & $\ldots$ & $\ldots$ & 364 & 333 \\
\hline
\end{tabular}

\begin{tabular}{|c|c|c|c|c|c|c|c|c|c|c|c|c|}
\hline \multicolumn{13}{|c|}{ Two companion } \\
\hline 2009 Nov & $L^{\prime}$ & $97.4 \pm 1.9$ & $2.4 \pm 1.2$ & $5.52 \pm 0.08$ & $\ldots$ & $\ldots$ & $\cdots$ & $85.3 \pm 2.4$ & $310.9 \pm 1.6$ & $5.53 \pm 0.07$ & 321 & 330 \\
\hline 2010 Aug & $L^{\prime}$ & $96.0 \pm 4.9$ & $350.1 \pm 3.4$ & $5.56 \pm 0.18$ & . & $\ldots$ & $\ldots$ & $83.0 \pm 4.1$ & $307.2 \pm 3.1$ & $5.33 \pm 0.13$ & 135 & 134 \\
\hline 2010 Nov & $L^{\prime}$ & $93.8 \pm 3.4$ & $356.7 \pm 2.4$ & $5.33 \pm 0.15$ & $\ldots$ & $\ldots$ & $\ldots$ & $85.7 \pm 3.8$ & $309.8 \pm 2.9$ & $5.26 \pm 0.11$ & 100 & 106 \\
\hline 2010 & $L^{\prime}$ & $94.9 \pm 2.9$ & $356.5 \pm 1.9$ & $5.46 \pm 0.12$ & & . & & $84.1 \pm 2.8$ & $310.0 \pm 2.0$ & $5.28 \pm 0.08$ & 238 & 246 \\
\hline $2009+2010$ & $L^{\prime}$ & $96.5 \pm 1.6$ & $1.0 \pm 1.1$ & $5.51 \pm 0.07$ & $\ldots$ & $\ldots$ & $\ldots$ & $84.6 \pm 1.9$ & $311.0 \pm 1.2$ & $5.45 \pm 0.06$ & 571 & 582 \\
\hline 2010 Nov & $K^{\prime}$ & $\ldots$ & $\ldots$ & $\ldots$ & $67.5 \pm 1.6$ & $333.3 \pm 1.3$ & $6.68 \pm 0.09$ & $88.9 \pm 2.6$ & $302.9 \pm 1.4$ & $7.20 \pm 0.13$ & 296 & 330 \\
\hline \multicolumn{13}{|c|}{ Three companion } \\
\hline 2009 Nov & $L^{\prime}$ & $112.4 \pm 6.4$ & $28.4 \pm 4.2$ & $6.35 \pm 0.21$ & $95.6 \pm 2.2$ & $357.4 \pm 2.7$ & $5.49 \pm 0.09$ & $85.1 \pm 2.6$ & $306.8 \pm 1.8$ & $5.55 \pm 0.08$ & 291 & 327 \\
\hline 2010 Aug & $L^{\prime}$ & $111.4 \pm 5.9$ & $13.7 \pm 3.8$ & $5.81 \pm 0.24$ & $92.1 \pm 4.9$ & $336.8 \pm 4.3$ & $5.36 \pm 0.15$ & $88.8 \pm 6.0$ & $295.0 \pm 5.0$ & $5.51 \pm 0.18$ & 113 & 131 \\
\hline 2010 Nov & $L^{\prime}$ & $91.5 \pm 4.1$ & $3.0 \pm 5.4$ & $5.51 \pm 0.14$ & $155.7 \pm 12.2$ & $323.5 \pm 2.9$ & $6.26 \pm 0.34$ & $85.8 \pm 4.8$ & $310.5 \pm 3.4$ & $5.25 \pm 0.11$ & 90 & 103 \\
\hline 2010 & $L^{\prime}$ & $104.7 \pm 4.7$ & $11.2 \pm 3.8$ & $5.81 \pm 0.19$ & $92.4 \pm 4.0$ & $337.3 \pm 4.7$ & $5.43 \pm 0.13$ & $86.6 \pm 4.2$ & $299.1 \pm 4.1$ & $5.51 \pm 0.15$ & 211 & 243 \\
\hline $2009+2010$ & $L^{\prime}$ & $106.8 \pm 3.2$ & $15.0 \pm 2.7$ & $5.89 \pm 0.14$ & $92.8 \pm 2.5$ & $344.7 \pm 3.6$ & $5.65 \pm 0.09$ & $87.4 \pm 2.2$ & $302.6 \pm 2.1$ & $5.56 \pm 0.08$ & 519 & 579 \\
\hline 2010 Nov & $K^{\prime}$ & $67.0 \pm 3.2$ & $12.3 \pm 2.8$ & $7.40 \pm 0.19$ & $64.4 \pm 1.5$ & $334.8 \pm 1.5$ & $6.59 \pm 0.09$ & $82.5 \pm 2.4$ & $302.3 \pm 1.5$ & $7.06 \pm 0.12$ & 265 & 327 \\
\hline \multicolumn{13}{|c|}{ Three (global) } \\
\hline ALL & $L^{\prime}$ & $100.7 \pm 1.9$ & $5.6 \pm 1.1$ & $5.69 \pm 0.08$ & $71.9 \pm 1.6$ & $335.2 \pm 1.3$ & $6.30 \pm 0.19$ & $88.2 \pm 1.8$ & $304.7 \pm 1.2$ & $5.73 \pm 0.09$ & 851 & 912 \\
\hline ALL & $K^{\prime}$ & $\ldots$ & $\ldots$ & $7.85 \pm 0.28$ & $\ldots$ & $\ldots$ & $6.73 \pm 0.10$ & $\ldots$ & $\ldots$ & $7.13 \pm 0.12$ & $\ldots$ & $\ldots$ \\
\hline
\end{tabular}

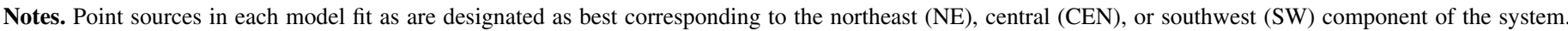

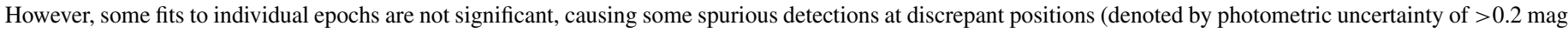
or $\mathrm{S} / \mathrm{N}<5$ ). All subsequent analysis uses the properties in the global fit.

resolved nature, would need to be a multiple system or a galaxy). The proper motion of $\mathrm{LkCa} 15$ reported by UCAC3

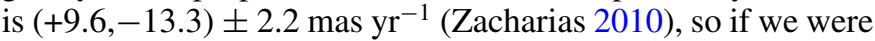
observing a background source or sources, then the $L^{\prime}$ detections should have moved by $(\Delta \alpha, \Delta \delta)=(+8.4,-11.6) \pm 1.9$ mas between the 2009 and 2010 epochs. The NE and CEN sources appear to yield low-quality fits at individual epochs, perhaps because flux is allowed to shift between the two nominal positions. However, the SW source appears fairly consistent between all epochs, and hence provides the best opportunity to measure the relative motion. We found that between 2009 (November) and 2010 (August plus November), the source position differed by $(\Delta \alpha, \Delta \delta)=(-7.5,-8.8) \pm 6.7$ mas. The disagreement with non-movement is therefore $16.2 \pm 7.0$ mas, or $2.3 \sigma$. Intriguingly, this motion is almost entirely in the P.A. direction, suggesting that we might be seeing orbital motion. We also note that the probability of such a chance alignment is extremely small; the Two Micron All Sky Survey (2MASS) Point Source Catalog lists only 77 sources with $K<15$ (and hence $L \lesssim 15$ ) within a radius of $<5$ arcmin of $\mathrm{LkCa} 15$, for an overall density of $3 \times 10^{-4}$ arcsec-2. The probability of finding one chance alignment within the disk gap of $\mathrm{LkCa} 15$
( $\rho \lesssim 300$ mas) is only $8 \times 10^{-5}$; even a survey of all $\sim 200$ stellar members of Taurus would have only a very small probability of finding a chance alignment.

Given the observed morphology of the $L^{\prime}$ and $K^{\prime}$ observations, then the LkCa 15 system appears to represent at least four sources: the primary, plus three sources of spatially resolved flux. We illustrate this geometry in Figure 3, where we show a multicolor RGB image with superpositions of an $L^{\prime}$ reconstruction based on all epochs (in red) and the $K^{\prime}$ reconstruction (in blue), along with submillimeter observations of the disk for context (Andrews et al. 2011). With this geometry in mind, we have simultaneously fit the observed closure phases at all epochs to obtain the most reliable characterization of these components (Table 2, bottom section: "Global Fit"). This fit indicates that there are detections of $K^{\prime}$ emission at the sites of the $L^{\prime}$ emission, and vice versa. However, since all of the sources are separated by the diffraction limit, then some caution is required; if the sources are more complex than simply three point sources, then a mismatch between the model and the observations could cause apparent flux to be transferred between the sources. 


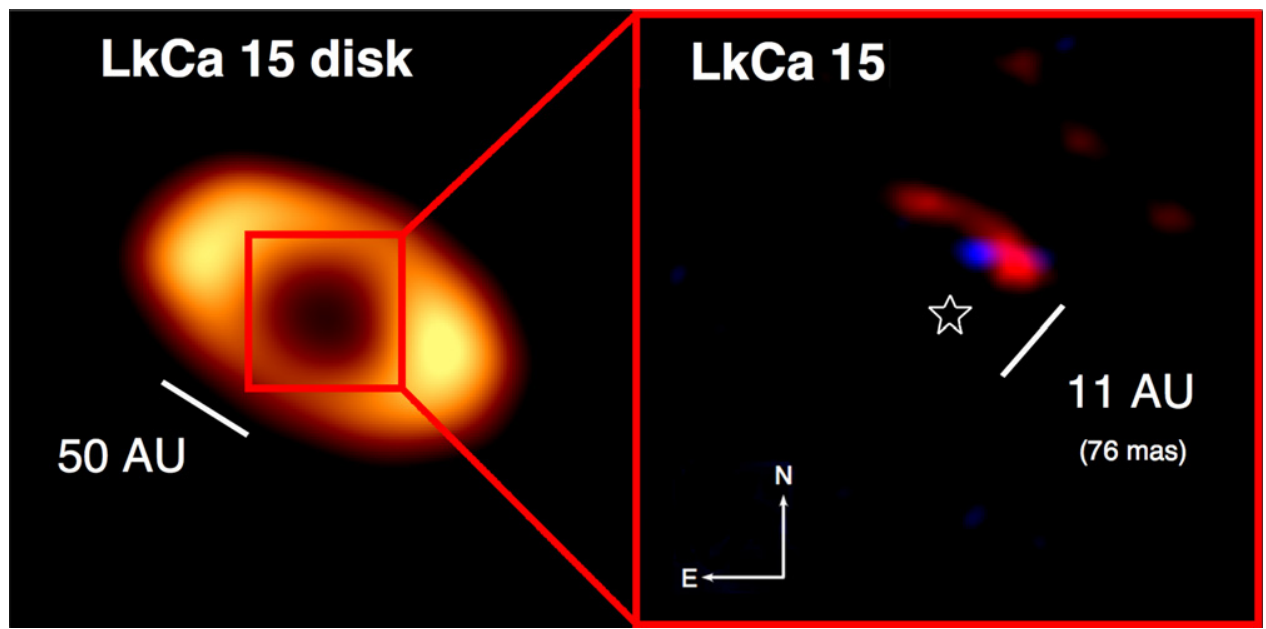

Figure 3. Left: the transitional disk around LkCa 15, as seen at a wavelength of $850 \mu \mathrm{m}$ (Andrews et al. 2011). All of the flux at this wavelength is emitted by cold dust in the disk; the deficit in the center denotes an inner gap with radius of $\sim 55 \mathrm{AU}$. Right: an expanded view of the central part of the cleared region, showing a composite of two reconstructed images (blue: $K^{\prime}$ or $\lambda=2.1 \mu \mathrm{m}$, from 2010 November; red: $L^{\prime}$ or $\lambda=3.7 \mu \mathrm{m}$, from all epochs) for LkCa 15 . The location of the central star is also marked. Most of the $L^{\prime}$ flux appears to come from two peaks that flank a central $K^{\prime}$ peak, so we model the system as a central star and three faint point sources.

\subsection{Orbital, Morphological, and Atmospheric Properties}

The observed morphology of LkCa 15's candidate companion is more complicated than that of older directly imaged exoplanets, which are seen as unresolved point sources (Marois et al. 2008; Kalas et al. 2008; Lagrange et al. 2009). The flux is mostly concentrated in a single unresolved location at $2.1 \mu \mathrm{m}$, but it is clearly extended at $3.7 \mu \mathrm{m}$. The most simple interpretation is that the central source is therefore a newly formed exoplanet, which emits significant flux at $2.1 \mu \mathrm{m}$ due to either a warm atmospheric temperature or accretion of hot material. The surrounding $3.7 \mu \mathrm{m}$ dominated emission would then trace extended circumplanetary material, most likely as it is accreting down to the planet, though perhaps as it accretes past the planet and onto the inner disk (e.g., Dodson-Robinson \& Salyk 2011). We can extrapolate the orbital radii, absolute magnitudes, and colors of these structures from our global fit of all observations (Table 2, bottom section) using the apparent magnitudes, distance, and age for $\mathrm{LkCa} 15$ which we describe further in Appendix.

We converted the observed separation and P.A. for each source into a deprojected orbital radius using the observed disk geometry $\left(i=49^{\circ}\right.$, P.A. $=241^{\circ}$; Andrews et al. 2011): $R_{\mathrm{NE}}=20.1 \pm 2.8 \mathrm{AU}, R_{\mathrm{CEN}}=15.9 \pm 2.1 \mathrm{AU}, R_{\mathrm{SW}}=$ $18.4 \pm 2.6$ AU. Model fits for disks typically vary by $\sim 5-10^{\circ}$ between different observations and models of the same targets, so we adopt a systematic uncertainty of $\pm 5^{\circ}$ in the inclination; combined with the distance uncertainty of $\pm 15 \mathrm{pc}$, the total uncertainty in deprojected radii is $\sim 15 \%$. Given deprojected orbital radii of $\sim 16-20 \mathrm{AU}$, then the corresponding orbital period and orbital motion around a solar-type star are $\sim 90$ years and $\sim 4$ deg year $^{-1}$. Our astrometric precision for the central source (i.e., the proposed planet itself) is $\sim 1.5$ (for its $K^{\prime}$ emission), so it is plausible that we could see orbital motion at the $3 \sigma$ level within the next 1-2 years. Orbit determinations for other high-contrast companions (such as GJ 802 B; Ireland et al. 2008) show that the astrometric errors predicted by NRM are typically valid. The $L^{\prime}$ astrometry for the SW source might already be showing orbital motion, since the offset between 2009 and 2010 is almost entirely in the P.A. direction and has a magnitude of $1.7 \sigma$. However, if the emission comes from a spatially resolved region, then it could be subject to two uncertainties. Since we are fitting a potentially resolved source as a point source, model mismatch could cause systematic astrometric errors. More seriously, if the emission comes from an extended dusty structure, then the centroid of the emission itself could change (with respect to that structure's position) over time. Even if the dust producing the $L^{\prime}$ emission is orbiting at a Keplerian velocity, the emission from different points in the structure might wax or wane. A conservative estimate of orbital motion should be based on at least several additional epochs, in order to determine the residuals around its apparent orbital velocity.

The observed contrasts can be converted into absolute magnitudes using the observed photometry for LkCa $15 \mathrm{~A}$ (Appendix) and the distance to Taurus-Auriga, $145 \pm 15 \mathrm{pc}$ (Torres et al. 2009); the combined absolute magnitude and color for all three components are $M_{L^{\prime}}=6.8 \pm 0.2 \mathrm{mag}$ and $K^{\prime}-L^{\prime}=1.7 \pm 0.2 \mathrm{mag}$. Young hot-start planets should have SEDs similar to L dwarfs, so assuming an approximate temperature of $1500 \mathrm{~K}$ and appropriate bolometric corrections (Leggett et al. 2002), then the corresponding bolometric luminosity is $L_{\text {bol }}=2 \times 10^{-3} L_{\odot}$, with an uncertainty of at least a factor of 2-3 (depending on the actual temperature).

Since the observed flux comes from spatially resolved structures and not a single point source, then the physical properties of each component must be considered individually. If the flux seen from the central source (near the $K^{\prime}$ peak) corresponds to the planet, then its brightness and color $\left(M_{K^{\prime}}=9.1 \pm 0.2\right.$; $K^{\prime}-L^{\prime}=0.98 \pm 0.22$ ) are more consistent with a photosphere than with warm dust. For ages of 1 Myr or 5 Myr (bracketing the $1 \sigma$ limits on the age of $\mathrm{LkCa} 15)$, then this brightness would naively be consistent with a mass of $6 M_{\text {Jup }}$ or $15 M_{\text {Jup }}$ according to the "hot start" models (Chabrier et al. 2000). However, if this planet is newly formed, then even the value for $1 \mathrm{Myr}$ might be an overestimate. Furthermore, the presence of significant circumplanetary material suggests that it is quite likely to be accreting, and current planet formation models suggest that a giant planet should intercept much of the disk mass that would otherwise accrete onto the central star (Lubow \& D'Angelo 2006; Machida et al. 2010), typically $\dot{M}=10^{-7}-10^{-9} M_{\odot} \mathrm{yr}^{-1}$ 
(Gullbring et al. 1998). For an accretion rate of $\dot{M}=$ $10^{-8} M_{\odot} \mathrm{yr}^{-1}$, the corresponding accretion luminosity would be $\sim 10^{-3} L_{\odot}$, assuming an emission temperature of $1500 \mathrm{~K}$ (consistent with the observed $K-L^{\prime}$ color), a planetary mass of $5 M_{\mathrm{Jup}}$, and a planetary radius of $<5 R_{\mathrm{Jup}}$. Accretion therefore could explain all of the observed luminosity, and since a reservoir of $55 M_{\text {Jup }}$ of material remains in the outer disk, then the proposed accretion rate of $10^{-8} M_{\odot} \mathrm{yr}^{-1}$ could be sustained for the entire $5 \mathrm{Myr}$ lifetime of a typical protoplanetary disk.

The emission from the surrounding material appears to be much redder (and hence cooler), with much more flux emitted at $3.7 \mu \mathrm{m}$ than at $2.1 \mu \mathrm{m}\left(M_{L^{\prime}}=7.5 \pm 0.2 \mathrm{mag}\right.$ and $K-L^{\prime}=2.7 \pm 0.3 \mathrm{mag}$ for NE, $M_{L]}=7.4 \pm 0.2 \mathrm{mag}$ and $K-L=1.94 \pm 0.16 \mathrm{mag}$ for $\mathrm{SW})$. This red color suggests that the material is quite cool $\left(T_{\text {eff }}<1000 \mathrm{~K}\right)$, though still much warmer than the ambient temperature at this distance from the star $(\sim 100 \mathrm{~K}$ for large dust grains; Section 3.4$)$. The presence of circumplanetary material is expected, since both the planet and inner disk should be accreting mass from the remaining protoplanetary disk (where a substantial reservoir of material remains, $55 M_{\mathrm{Jup}}$ ). However, the material is much more spatially extended than models have predicted (Machida et al. 2010). Our observations place the emitting material at $6 \pm 1 \mathrm{AU}$ away from the central source, which is larger than the Hill radius for a $10 M_{\text {Jup }}$ object (2-3 AU) and significantly larger than the radius at which material heated by the planet should be emitting in the NIR ( $\ll 1 \mathrm{AU})$. Conversely, the distance is too small for the flanking components to represent material accumulating at the Trojan points. These two components are separated from the central component by only $20^{\circ} \pm 3^{\circ}$ in the deprojected plane of the disk, whereas the Trojan points should lead and trail a planet by $60^{\circ}$.

Given the luminosity of $\mathrm{LkCa} 15$, micron-sized dust grains at an orbital radius of $\sim 25$ AU should have an equilibrium temperature of only $100 \mathrm{~K}$, which is too cool to produce significant flux at $3.7 \mu \mathrm{m}$. This suggests that energy is being generated (or delivered) to the extended circumplanetary environment in some other way. The direct accretion luminosity of the planet is three orders of magnitude smaller than the luminosity of LkCa $15 \mathrm{~A}$, so direct irradiation by the planet is also insufficient. Another plausible explanation is the deposition of orbital kinetic energy as material accretes into the circumplanetary environment from the outer disk. The expected accretion rate and typical orbital velocities at that radius from the star are large enough to deliver the needed energy, but detailed modeling will be needed to determine if material will be heated sufficiently as to emit in the NIR. Finally, one possible explanation is that the energy is transported out from the planet by accretion jets or winds, as a significant fraction of accreting material should be launched back outward from the planet (Herbig 1950; Haro 1952; Konigl \& Pudritz 2000; Shu et al. 2000). If this higher-velocity material impacts the complex circumplanetary environment, perhaps guided by the global magnetic field of the disk, then it could deposit sufficient energy to heat that environment. More rigorous testing of these models will required additional observations at shorter or longer wavelengths (in order to refine the temperature estimates for each spatially resolved component) or direct identification of the circumplanetary dust distribution with observations from ALMA.

In Figure 4, we show the brightness and color of the individual components and the full structure as compared to free-floating stars and brown dwarfs within the Taurus star-forming region (Luhman et al. 2010). The observed fluxes for any individual

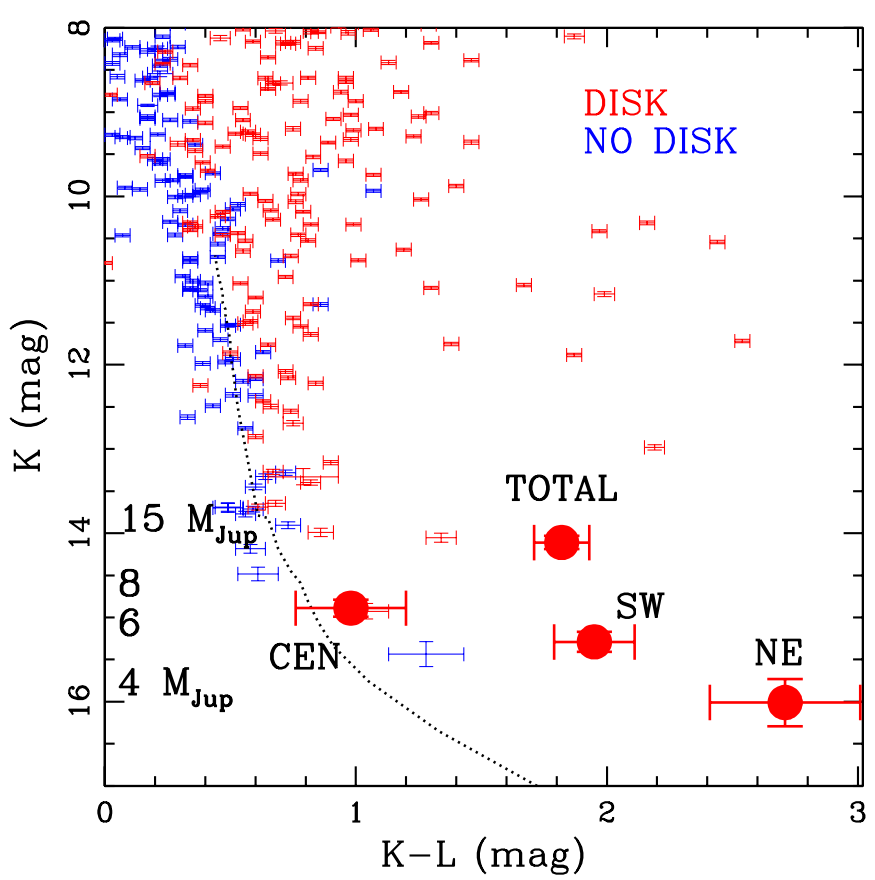

Figure 4. $(K, K-L)$ color-magnitude diagram for Taurus, showing all free-floating members of Taurus (open error bars; Luhman et al. 2010), our measurements for the three distinct morphological segments of the resolved structures (labeled NE, CEN, and SW), and the combined color and magnitude of all three (TOTAL). Taurus members without disks are shown in blue, and tend to follow the 1 Myr theoretical isochrone of the Lyon models (dotted line; Chabrier et al. 2000). Objects with circumstellar disks tend to be redder than those without. The central source also falls near the $1 \mathrm{Myr}$ isochrone, suggesting that it could represent the actual planet; if so, the inferred mass is $\sim 6 \pm 1 M_{\text {Jup }}$. However, the luminosity for this source could be dominated by accretion luminosity and hence not indicative of the true planetary mass.

(A color version of this figure is available in the online journal.)

component, or even for the sum, are fainter than all but the few least-massive members of Taurus-Auriga, which themselves fall in the planetary-mass range (e.g., Luhman et al. 2009). If other explanations can be rejected, then this low luminosity strongly suggests that our observations have revealed a planetary companion.

\subsection{Heating Processes for the Extended Circumplanetary Material}

We interpret our observations to represent a single planet (with a relatively neutral near-IR color) surrounded by resolved circumplanetary material (with a very red near-IR color). The modest color of the planet is not surprising, since its brightness is likely dominated by accretion luminosity that should be much bluer than the underlying photosphere. However, the brightness and size of the circumplanetary region requires additional explanation. As we discuss further in Section 3.4, material should only glow in the NIR if it has a temperature of $\gtrsim 500-1000 \mathrm{~K}$; otherwise, its blackbody peak shifts to much longer wavelengths. Even for relatively luminous stars, dust is only heated to this temperature within the inner $\sim 0.1-0.2$ AU (Olofsson et al. 2011). We therefore must question if radiative heating from a planet is sufficient. Given the $K^{\prime}$ magnitude and $K^{\prime}-L^{\prime}$ color of the proposed planet, then its total luminosity is $L \sim 10^{-3} L_{\odot}$. Large dust grains should absorb this incident flux in proportion to their cross-section and emit it in proportion to their surface area, so their equilibrium temperature depends only on the distance from the planet. At a distance of $\sim 5 \mathrm{AU}$, 
the equilibrium temperature for large dust grains should be $\sim 20-25 \mathrm{~K}$, a factor of 20 too low to explain the observed $L^{\prime}$ flux.

This energy could also represent shock-heating of circumplanetary material due to the deposition of orbital kinetic energy from disk material into the circumplanetary environment. The typical orbital velocity at an orbital radius of $\sim 20 \mathrm{AU}$ from $\mathrm{LkCa} 15 \mathrm{~A}$ should be $\sim 7 \mathrm{~km} \mathrm{~s}^{-1}$, and the kinetic energy of accreted disk material could be liberated as thermal energy if it impacts on circumplanetary material moving with a similar speed, but in a different direction. If the typical accretion rate is $\sim 10^{-7}-10^{-8} M_{\odot} \mathrm{yr}^{-1}$, then the corresponding luminosity will be $\sim 10^{-3}-10^{-4} L_{\odot}$. The high end of this range is consistent with the observed luminosity, so this explanation could be feasible. However, the observed temperature of such material will depend on the detailed physics of the shock-heating process, and hence will require more sophisticated modeling and analysis. Furthermore, the cooling timescale for small dust grains is very short $(<1 \mathrm{~s})$, so the similarity between our 2009 and 2010 observations suggests that this heating is a continuous process and not the result of a single energetic event (such as a collisional cascade following a collision of large planetesimals).

Another plausible explanation is that we are indeed seeing energy from the final accretion of material onto a central planet, but it is being transported to the observed sites ( $~ 5$ AU away from the planet) through some process other than direct radiation that is not attenuated by a factor of $r^{-2}$. Accretion onto young stars is inextricably tied to the launching of jets (Herbig 1950; Haro 1952) and winds (Konigl \& Pudritz 2000; Shu et al. 2000), and those winds and jets can carry a significant amount of the accreted material outward at a high velocity. If planetary accretion also feeds such outflows, then this high-speed material could impact cool material in the surrounding circumplanetary environment and transform its kinetic energy into thermal energy, forming planetary equivalents of the bow shocks observed around stellar outflows. However, this explanation invokes even more open topics of star and planet formation than the previous hypothesis, so it also must await testing with more sophisticated modeling.

Finally, a consistent (but improbable) explanation for the geometry might be that we are seeing two extremely red planets, and that the central blue feature represents a non-planetary feature. Given the extreme youth of the system, it is plausible that a stable orbital solution exists for two similar-luminosity (and hence similar-mass) planets to share nearly the same orbital radius for a sufficient length of time. If these planets were locked into a 1:1 resonance, then they might also support a quasistable point (equivalent to Trojan points) midway between them. If dust accumulated at this quasistable point, then it would reflect light from the central star with approximately the stellar color (Duchêne et al. 2004), matching the observed color of the central source. The most expedient way to rule out this improbable solution is to widen the wavelength coverage of our observations (i.e., to $H$ or $M^{\prime}$ ), which should distinguish intrinsically cool planets from intrinsically blue reflected light.

\subsection{Rejected Alternative Explanations}

We also must weigh alternative explanations for our observations. As we discussed in Section 3.1, the consistent astrometry across a one-year baseline suggests that our discovery is astrophysical and is comoving. One possibility is that the structure could also be caused by a more massive companion that is obscured at the wavelength of our observations, and hence made to look fainter. Since LkCa 15 is surrounded by a dusty disk, then we could also have observed thermal emission from dust that is heated by the primary or directly reflected light from the primary that is incident on the disk. Finally, we could be observing line emission from the gas (i.e., $\mathrm{Br} \gamma$ ) or from polycyclic aromatic hydrocarbons (PAHs).

The purported planetary companion could represent an unseen binary companion, perhaps obscured by an edge-on disk so that it appears significantly fainter. The example of $\mathrm{CoKu}$ Tau/4 demonstrates that "transitional disks" can host binary companions (Ireland \& Kraus 2008), and other binary systems, like HK Tau and HV Tau, are known to have wider components which are obscured in this way (Stapelfeldt et al. 1998, 2003). However, most close binary systems are thought to have coplanar disks (Jensen et al. 2004), so any disk around this putative stellar companion should not produce significant obscuration along our line of sight. The observed geometry also is inconsistent with that seen for edge-on disk systems like HV Tau (Duchêne et al. 2010), where the central dust lane also separates the flux into separate lobes, but those lobes are more widely separated at shorter wavelengths (due to the higher extinction through the disk near the midplane). Finally, if the putative companion was obscured by a massive circumstellar disk, then it should produce significant mid-infrared and submillimeter continuum emission that would be distinguished from the outer disk (Espaillat et al. 2007; Andrews et al. 2011).

We also must consider the prospect that the flux we attribute to a planet could instead represent energy from the central star that is being processed and re-emitted by the disk. The central hole is thought to be largely cleared of disk material, but it is plausible that grain growth and settling could instead cause the low apparent optical depth for submillimeter photons. However, at orbital radii of $\sim 20 \mathrm{AU}$ from a star with $T_{*}=4000 \mathrm{~K}$ and a stellar radius of $\sim 2 R_{\odot}$, the equilibrium temperature is only $\sim 100 \mathrm{~K}$, such that the blackbody peak is at $\sim 30 \mu \mathrm{m}$; emission in the $L^{\prime}$ filter would be negligible in comparison. This simple calculation is consistent with more complex models of disk structure and evolution, which find that only the innermost regions reach temperatures of $>500 \mathrm{~K}$ (D'Alessio et al. 2006). In fact, for a full optically thick disk, the majority of light that is processed and re-emitted in the near-infrared comes from the inner $\sim 0.1 \mathrm{AU}$ of the disk (Olofsson et al. 2011). Even inner gaps with a size of $\sim 1$ AU lead to an observed paucity of flux at $<10 \mu \mathrm{m}$ (Najita et al. 2010). Small dust grains can be much warmer than large dust grains, but even they cannot achieve the necessary temperature. Where dust has an opacity proportional to $\lambda^{-1}$, the dust temperature is given by

$$
T_{d}=\left(r_{d} / 2 R_{*}\right)^{2 / 5} T_{*} .
$$

Hence, the dust temperature could be as high as $246 \mathrm{~K}$. With an optical depth of unity at the Planck peak of $700 \mathrm{~nm}$ and a $25 \mathrm{AU}^{2}$ cross-sectional area (the maximum allowed by the resolution of Keck), even this extreme dust could not provide sufficient flux to match the point sources we model in $L$-band. Furthermore, this hypothetical dust would shadow the outer disk, which is not observed, and it cannot explain the $K^{\prime}$ morphology.

Another possibility is that we are seeing light from the disk, but rather than thermal emission from heated material, it is direct reflection of incident light from the central star. Reflected light has been cited as the likely explanation for the ring of circumstellar light previously reported in $H$ band (Thalmann et al. 2010), which is coincident with the $~ 50 \mathrm{AU}$ 
radius of the inner disk edge suggested by SED modeling (Espaillat et al. 2007, 2010) and directly observed in submm emission (Andrews et al. 2011). The disk geometry suggested by the $H$ band emission somewhat favors placing the northwest face of the disk in the background, with a nearly normal incidence of reflection off the face of the disk wall at $\sim 50 \mathrm{AU}$. If there were another wall located at a radius of $\sim 20 \mathrm{AU}$, then it could reflect light in a similar manner. However, this explanation faces a significant challenge. Any inner wall at smaller separations must reflect $\sim 1.5 \%$ of the incident $L^{\prime}$ stellar flux from an arc of $\sim 60^{\circ}$, or $\sim 10 \%$ of the incident flux in a complete circle around the star. The wall height needed to intercept this amount of flux ( $\sim 5 \mathrm{AU})$ would shadow the wall at $50 \mathrm{AU}$, an effect that is not seen in the SED fits or the reflected light. This explanation might still be feasible if a wall at $25 \mathrm{AU}$ were optically thick at $L^{\prime}$, but optically thin at optical wavelengths (allowing the majority of the stellar flux to pass). However, this type of opacity law is very much contrary to all standard dust types, which are either gray or have opacity increasing to shorter wavelengths (Schlegel et al. 1998).

If the disk geometry were reversed, placing the northwest face in the foreground, then our reported detection could also represent forward-scattered light from the central star, which is being reflected back to our line of sight by material inside the disk. As for the previous case, we must give this explanation extra merit since we have detected the spatially resolved flux near the minor axis of the disk, as we would expect from forward scattering. However, the same drawbacks also apply, in that any material must reflect sufficient light at $K^{\prime}$ and $L^{\prime}$ to explain our detection, but still remain optically thin in the optical. Furthermore, the extremely red color of the light is also difficult to explain with forward scattering. Most observations of disks in scattered light, such as for the circumbinary ring of GG Tau (Duchêne et al. 2004), find colors which are neutral or moderately blue. The extremely red color of our detection is not consistent with these other observations. Even if the dust grain size distribution were chosen to optimally redden the $K^{\prime}-L^{\prime}$ color (i.e., a single-sized grain population with a radius of $\sim 1.5 \mu \mathrm{m})$, then it could only redden the reflected light by $\Delta\left(K^{\prime}-L^{\prime}\right) \sim 0.5$ (to $K^{\prime}-L^{\prime} \sim 1.5$ ), whereas the red sources have an observed color of $K^{\prime}-L^{\prime}=2.0-2.7$.

Finally, we must consider the possibility that we are not observing continuum emission from a planetary companion and circumplanetary dust, but instead are seeing line emission from gas (via $\mathrm{Br} \gamma$ at $2.16 \mu \mathrm{m}$ ) and PAHs (at $3.3 \mu \mathrm{m}$ ). However, the blue edge of the $L^{\prime}$ filter is located at $3.43 \mu \mathrm{m}$, so only the extreme red wing of the PAH line might pass any light through this broadband filter. Furthermore, if all of the flux observed were line emission, then given the apparent broadband flux ratios $\left(\Delta K^{\prime}=6.7\right.$ and $\left.\Delta L^{\prime}=4.7\right)$ and the ratio of the line width to the width of the full broadband filter (e.g., $\gg 100: 1$ for $\mathrm{Br} \gamma$ ), the line emission should exceed the continuum and be observed clearly in spectra of the entire system. Previous observations at 2-5 $\mu \mathrm{m}$ have seen no evidence of such line emission (Espaillat et al. 2008). Nonetheless, such emission would produce a large spectroastrometric signal (e.g., Pontoppidan et al. 2011), so such an observation should be attempted to conclusively rule out this explanation.

\section{IMPLICATIONS FOR PLANET FORMATION AND EVOLUTION}

The apparent planetary companion to $\mathrm{LkCa} 15$ is the first likely exoplanet to be discovered at its time of formation, and hence it provides a new view of planet formation and early planet evolution. Planet formation models make unique predictions regarding the location and epoch of planet formation (Pollack et al. 1996; Boss 2001; Ida \& Lin 2004), so the orbital radius of the planet and the age of its parent star provide the first direct evidence for distinguishing between these models. Also, evolutionary models make extremely discrepant predictions regarding the luminosity of planets as a function of age (Chabrier et al. 2000; Fortney et al. 2008), though this discussion is complicated by possible flux contributions from circumplanetary material and accretion luminosity.

If the planet is coplanar with its disk, then the current orbital radius is $15.7 \pm 2.1 \mathrm{AU}$. The core accretion model of planet formation is primarily limited by the ability to assemble a $20 M_{\oplus}$ core, so it is thought to form planets much more efficiently near the snow line $(a \sim 3-5 \mathrm{AU})$ than at these larger radii (Tsiganis et al. 2005; Kennedy \& Kenyon 2008). Recent models of in situ formation suggest that a Saturn analog could potentially form at $a \sim 10$ AU within 3-4 Myr (Dodson-Robinson et al. 2008), but for Uranus and Neptune analogs to be formed within $<5 \mathrm{Myr}$, they must begin at $a<15 \mathrm{AU}$ and then subsequently migrate outward (Dodson-Robinson \& Bodenheimer 2010; Bromley \& Kenyon 2011). In contrast, disk instability models are more efficient at forming planets at larger radii $(a>25-50 \mathrm{AU}$; Rafikov 2005; Boley 2009; Meru \& Bate 2010; Boss 2011) since they are primarily limited by the ability of gas to efficiently cool and by the shear of differential rotation speeds, both of which limit the ability for a protoplanetary clump to exceed the Toomre stability criterion (Toomre 1964). The large orbital radius is therefore more consistent with formation via disk instability, though it is unclear why fragmentation occurred at a radius of $20 \mathrm{AU}$, rather than in the massive, cold outer disk. It is possible that orbital migration has already occurred, and hence planet formation occurred at either larger or smaller radii.

The implications of the age of $\mathrm{LkCa} 15$ are not as clear. A comparison to stellar evolutionary models suggests that the most likely age for $\mathrm{LkCa} 15 \mathrm{~A}$ is $2 \mathrm{Myr}$, with a $1 \sigma$ range of 1-4 Myr (Kraus \& Hillenbrand 2009). As we discussed above, even formation at $a<15 \mathrm{AU}$ seems to require at least 2-3 Myr for core accretion models, so they would be more plausible if the primary star were to fall at the older end of this allowed range. Formation via disk instability does not necessarily carry a strong age constraint, as the collapse interval is thought to be quite short. However, disk instability should be most likely at young ages, when the disk is most massive. It is unclear why a planet would only form after several Myr, when part of the disk mass has already accreted to the star, so if the planet is forming via disk instability then the star should fall at the younger end of the allowed age range. The distinction between these possibilities would grow stronger if the age of $\mathrm{LkCa} 15$ could be determined more precisely, such as by refining its temperature (and hence its position on the HR diagram). However, systematic uncertainties in the ages of young stars probably limit any such determinations until the models can be better calibrated (Hillenbrand \& White 2004).

The distance between the planet and the inner disk edge is also somewhat at odds with theoretical expectations. When a planet clears a gap in its disk, then the size of the gap should be approximately equal to the planet's Hill radius (Crida et al. 2006); even if the planet is $\sim 10 M_{\text {Jup }}$, then its Hill radius is only $\sim 2-3$ AU. However, models of the disk SED and direct 
submillimeter observations of the dust distribution show that the inner edge of the disk has a radius of $\sim 55 \mathrm{AU}$ (Espaillat et al. 2007; Andrews et al. 2011), which is $>25$ AU outside of the planet's current orbital radius. This discrepancy could indicate that there are other, less luminous planets in the system and we simply cannot detect them yet, a possibility that has been suggested to explain the wide cleared gaps for many transitional disks (e.g., Zhu et al. 2011; Dodson-Robinson \& Salyk 2011). However, it is also possible that the planet is on an eccentric orbit, and hence the radius of the inner disk edge is set by the apastron distance. This assertion will be difficult to test since the planet's likely orbital period is so long ( $\sim 90 \mathrm{yr}$ for a circular orbit), making a full orbit fit difficult for at least several decades. However, scattered-light observations of the disk's inner edge have shown that it is off-center compared to the primary star, which is often a sign of perturbation by a body on an eccentric orbit (Thalmann et al. 2010). Additional modeling of the planet-disk interaction could provide constraints on the most likely orbit for the planet.

Finally, the luminosity also provides a crucial first data point for calibrating the brightness evolution of young giant planets. Models that include realistic simulations of the core accretion process (the "cold start" models; Marley et al. 2007; Fortney et al. 2008) suggest that even young exoplanets should not be more luminous than $\sim 10^{-5} L_{\odot}$. By contrast, models which assume a higher-entropy initial state such as for collapse out of a gas cloud (the "hot start" models; Chabrier et al. 2000) predict that young planets could be as bright as $\sim 10^{-3} L_{\odot}$. The spatially extended morphology that we observe makes it difficult to infer a strong measurement of the total luminosity for the planet, but as we show in Section 3.2, the observed flux ratio corresponds to a luminosity of $\sim 10^{-3} L_{\odot}$. This luminosity falls at the top end of the range permitted by the "hot start" models, and several orders of magnitude higher than the estimates of the "cold start" models. The same result has been found for moderately older direct-imaged exoplanets, such as Beta Pic b (Lagrange et al. 2009; Quanz et al. 2010; Currie et al. 2011a) and HR 8799 bcde (Marois et al. 2008, 2010; Bowler et al. 2010; Currie et al. 2011b; Madhusudhan et al. 2011). However, newer models suggest that the luminosities of young planets might be intermediate between these extremes, making this distinction less clear (e.g., Spiegel \& Burrows 2011).

However, there is a strong caveat regarding the luminosity we infer for LkCa 15's planetary companion. Since our survey is targeting planets in transition disks, then by definition, we have observed this apparent planet at a point where its luminosity should be maximized. A gap in the disk should only form once a gas giant planet has a significant envelope, which only happens when it is accreting a significant amount of the mass crossing its orbit into the inner disk. Observations of other young solartype stars show that material in their disks will typically move inward at an accretion rate of $\sim 10^{-8} M_{\odot} \mathrm{yr}^{-1}$ (Gullbring et al. 1998); the planet is therefore likely to be accreting material very quickly, and hence generating a significant accretion luminosity. Realistic values for the planet mass/radius and accretion rate yield accretion luminosities as high as $\sim 10^{-3} L_{\odot}$ (Section 3.2), matching our inferred value. This brief period of high luminosity is seen as a luminosity spike in the "cold start" models, which find that even a $\sim 1 M_{\text {Jup }}$ planet could reach a peak luminosity of $\sim 5 \times 10^{-3} L_{\odot}$ at the epoch of peak growth. It therefore seems plausible that we are not seeing any flux from the planetary atmosphere itself, but merely the accretion excess that is being released by its rapid assembly.
The blue color of the central source and the red color of the surrounding material further supports this view. If the planet is rapidly accreting mass from the broader circumstellar environment, then it should be enshrouded in material that is in the final stages of accretion. For a free-floating brown dwarf, this material organizes itself into a disk (Scholz et al. 2007). However, those free-floating objects only accrete at a rate of $\sim 10^{-13} M_{\odot} \mathrm{yr}^{-1}$ (Herczeg et al. 2009), which is five orders of magnitude lower. It is unclear whether the same geometry could be sustained with so much mass being continuously added to the environment. It seems equally probable that the accretion would take place via a very inflated disk or through an unexpected geometry. If so, then the very red color of the surrounding material should only be expected. The central planet would appear relatively blue due to accretion, but our expectations for the surrounding environment are largely unconstrained by theory.

\section{SUMMARY}

We have reported the direct-imaging discovery of a likely (proto)planet around the young transitional disk host $\mathrm{LkCa} 15$, located at the middle of the known gap in its disk. Our observations have revealed a faint and relatively blue point source, surrounded by co-orbital emission that is red and resolved into at least two sources. The most likely geometry consists of a newly formed gas giant planet that is surrounded by dusty material, and which has been caught at its epoch of formation. This discovery is the first direct evidence that at least some transitional disks do indeed host newly formed (or forming) exoplanetary systems, and the observed properties provide crucial insight into the gas giant formation process.

Additional studies of this system, both theoretical and observational, will be necessary to more fully understand its complicated colors and morphology, and ultimately to confirm its planetary nature. Broadband photometry at additional wavelengths should be feasible with existing instruments and will extend our knowledge of the spatially resolved broadband SED. Next-generation instruments like the Gemini Planet Imager will also be capable of observing LkCa 15 with NRM, and they will yield low-resolution spectra that show any influence of broad molecular absorption bands due to water or methane. Finally, submillimeter/millimeter observations with ALMA will directly track the dust in the $\mathrm{LkCa} 15$ system with sufficient sensitivity and resolution to distinguish the circumplanetary material, resolving any remaining ambiguities as to its spatial distribution and mass.

We thank Sean Andrews, Gregory Herczeg, Michael Liu, Jonathan Williams, Lucas Cieza, Lynne Hillenbrand, and Peter Tuthill for helpful discussions regarding this manuscript. We especially thank Sylvestre Lacour for comparison of some results of our pipeline with his pipeline and for offering helpful advice. We also thank the referee for an insightful critique that identified several improvements in the analysis. Finally, we thank Swinburne University of Technology for the use of their remote observing facilities. A.L.K. has been supported by NASA through Hubble Fellowship grant 51257.01 awarded by STScI, which is operated by AURA, Inc., for NASA under contract NAS 5-26555. We recognize and acknowledge the very significant cultural role and reverence that the summit of Mauna Kea has always had within the indigenous Hawaiian community. We are most fortunate to have the opportunity to conduct observations from this mountain. 


\section{APPENDIX}

\section{THE PROPERTIES OF LkCa 15 A}

The young solar analog $\mathrm{LkCa} 15$ is a $\mathrm{K} 7$ star located in the nearby $(145 \pm 15 \mathrm{pc})$ Taurus-Auriga star-forming region (Kenyon \& Hartmann 1995). Based on its position in the HR diagram, it has an age of $2_{-1}^{+2} \mathrm{Myr}$ (Kraus \& Hillenbrand 2009). The primary star's mass, $0.97 \pm 0.03 M_{\odot}$, has been measured from the rotation curve of the circumstellar disk (Simon et al. 2000). It has an observed brightness of $m_{[3.6]}=7.61 \pm 0.05 \mathrm{mag}$ in the IRAC $3.6 \mu \mathrm{m}$ filter, and thus is of similar brightness in the $L^{\prime}$ band (Rebull et al. 2010). According to 2MASS, its brightness is $K=8.16 \pm 0.02$ mag (Skrutskie et al. 2006). Given the well-known variability of young stars, the real uncertainty is probably at least $\sim 0.1 \mathrm{mag}$ in each filter (Carpenter et al. 2002). The extinction to LkCa 15 is negligible for the purposes of NIR observations, $A_{V}<1$ or $A_{K}<0.1$ (Kenyon \& Hartmann 1995).

We previously observed LkCa 15 with NRM in the $K^{\prime}$ band $(\lambda=2.1 \mu \mathrm{m})$ to determine if this cleared region could indicate the presence of a binary companion, but found no companions with contrast $\Delta K \leqslant 6.2 \mathrm{mag}\left(M>12 M_{\text {Jup }}\right)$ at a confidence level of $99.9 \%$ or $3.3 \sigma$ (Kraus et al. 2008). We have reanalyzed our $K^{\prime}$-band data for $\mathrm{LkCa} 15$ in order to place a more strict upper limit on the brightness of a companion at the known position from our $L^{\prime}$ band detections. Our new analysis suggests a stronger limit of $\Delta K<6.6 \mathrm{mag}$, suggesting that our old dataset nearly detected the $K^{\prime}$-band counterpart that we observed in 2010 November.

\section{REFERENCES}

Andrews, S., Wilner, D., Espaillat, C., et al. 2011, ApJ, 732, 42

Andrews, S. M., \& Williams, J. P. 2005, ApJ, 631, 1134

Boley, A. C. 2009, ApJ, 695, L53

Boss, A. P. 2001, ApJ, 563, 367

Boss, A. P. 2011, ApJ, 731, 74

Bowler, B. P., Liu, M. C., Dupuy, T. J., \& Cushing, M. C. 2010, ApJ, 723, 850 Bromley, B. C., \& Kenyon, S. J. 2011, ApJ, 731, 101

Brown, J. M., Blake, G. A., Qi, C., et al. 2009, ApJ, 704, 496

Calvet, N., D'Alessio, P., Watson, D. M., et al. 2005, ApJ, 630, L185

Carpenter, J. M., Hillenbrand, L. A., Skrutskie, M. F., \& Meyer, M. R. 2002, AJ, 124, 1001

Chabrier, G., Baraffe, I., Allard, F., \& Hauschildt, P. 2000, ApJ, 542, 464

Crida, A., Morbidelli, A., \& Masset, F. 2006, Icarus, 181, 587

Currie, T., Burrows, A., Itoh, Y., et al. 2011b, ApJ, 729, 128

Currie, T., Thalmann, C., Matsumura, S., et al. 2011a, ApJ, 736, L33

D’Alessio, P., Calvet, N., Hartmann, L., Franco-Hernández, R., \& Servín, H. 2006, ApJ, 638, 314

Dodson-Robinson, S. E., \& Bodenheimer, P. 2010, Icarus, 207, 491

Dodson-Robinson, S. E., Bodenheimer, P., Laughlin, G., et al. 2008, ApJ, 688, L99

Dodson-Robinson, S. E., \& Salyk, C. 2011, ApJ, 738, 131

Duchêne, G., McCabe, C., Ghez, A. M., \& Macintosh, B. A. 2004, ApJ, 606, 969

Duchêne, G., McCabe, C., Pinte, C., et al. 2010, ApJ, 712, 112

Espaillat, C., Calvet, N., D'Alessio, P., et al. 2007, ApJ, 670, L135

Espaillat, C., Calvet, N., Luhman, K. L., Muzerolle, J., \& D’Alessio, P. 2008, ApJ, 682, L125

Espaillat, C., D’Alessio, P., Hernández, J., et al. 2010, ApJ, 717, 441

Fortney, J. J., Marley, M. S., Saumon, D., \& Lodders, K. 2008, ApJ, 683, 1104 Gullbring, E., Hartmann, L., Briceno, C., \& Calvet, N. 1998, ApJ, 492, 323

Haro, G. 1952, ApJ, 115, 572

Herbig, G. H. 1950, ApJ, 111, 11

Herczeg, G. J., Cruz, K. L., \& Hillenbrand, L. A. 2009, ApJ, 696, 1589

Hillenbrand, L. A. 2008, Phys. Scr. T, 130, 014024

Hillenbrand, L. A., \& White, R. J. 2004, ApJ, 604, 741
Hinkley, S., Carpenter, J. M., Ireland, M. J., \& Kraus, A. L. 2011, ApJ, 730 , L21

Huelamo, N., Lacour, S., Tuthill, P., et al. 2011, A\&A, 528, L7

Ida, S., \& Lin, D. N. C. 2004, ApJ, 616, 567

Ireland, M. J., Kraus, A., Martinache, F., Lloyd, J. P., \& Tuthill, P. G. 2008, ApJ, 678,463

Ireland, M. J., \& Kraus, A. L. 2008, ApJ, 678, L59

Ireland, M. J., Monnier, J. D., \& Thureau, N. 2006, Proc. SPIE, 6268, 62681T

Jensen, E. L. N., Mathieu, R. D., Donar, A. X., \& Dullighan, A. 2004, ApJ, 600, 789

Kalas, P., Graham, J. R., Chiang, E., et al. 2008, Science, 322, 1345

Kennedy, G. M., \& Kenyon, S. J. 2008, ApJ, 673, 502

Kenyon, S. J., \& Hartmann, L. 1995, ApJS, 101, 117

Konigl, A., \& Pudritz, R. E. 2000, in Protostars and Planets IV, ed. V. Mannings, A. P. Boss, \& S. S. Russell (Tucson, AZ: Univ. Arizona Press), 759

Kraus, A. L., \& Hillenbrand, L. A. 2009, ApJ, 704, 531

Kraus, A. L., Ireland, M. J., Martinache, F., \& Hillenbrand, L. A. 2011, ApJ, 731,8

Kraus, A. L., Ireland, M. J., Martinache, F., \& Lloyd, J. P. 2008, ApJ, 679, 762

Lacour, S., Tuthill, P., Amico, P., et al. 2011, A\&A, 532, A72

Lafrenière, D., Marois, C., Doyon, R., Nadeau, D., \& Artigau, É. 2007, ApJ, 660,770

Lagrange, A., Gratadour, D., Chauvin, G., et al. 2009, A\&A, 493, L21

Leggett, S. K., Golimowski, D. A., Fan, X., et al. 2002, ApJ, 564, 452

Lubow, S. H., \& D’Angelo, G. 2006, ApJ, 641, 526

Luhman, K. L., Allen, P. R., Espaillat, C., Hartmann, L., \& Calvet, N. 2010, ApJS, 186, 111

Luhman, K. L., Mamajek, E. E., Allen, P. R., \& Cruz, K. L. 2009, ApJ, 703, 399

Machida, M. N., Kokubo, E., Inutsuka, S., \& Matsumoto, T. 2010, MNRAS, 405,1227

Madhusudhan, N., Burrows, A., \& Currie, T. 2011, ApJ, 727, 34

Marley, M. S., Fortney, J. J., Hubickyj, O., Bodenheimer, P., \& Lissauer, J. J. 2007, ApJ, 655, 541

Marois, C., Macintosh, B., Barman, T., et al. 2008, Science, 322, 1348

Marois, C., Zuckerman, B., Konopacky, Q. M., Macintosh, B., \& Barman, T. 2010, Nature, 468, 1080

Martinache, F. 2010, ApJ, 724, 464

Meru, F., \& Bate, M. R. 2010, MNRAS, 406, 2279

Monnier, J. D., Zhao, M., Pedretti, E., et al. 2007, Science, 317, 342

Najita, J. R., Carr, J. S., Strom, S. E., et al. 2010, ApJ, 712, 274

Nakajima, T., Kulkarni, S. R., Gorham, P. W., et al. 1989, AJ, 97, 1510

Olofsson, J., Benisty, M., Augereau, J.-C., et al. 2011, A\&A, 528, L6

Pauls, T. A., Young, J. S., Cotton, W. D., \& Monnier, J. D. 2005, PASP, 117, 1255

Pollack, J. B., Hubickyj, O., Bodenheimer, P., et al. 1996, Icarus, 124, 62

Pontoppidan, K. M., Blake, G. A., \& Smette, A. 2011, ApJ, 733, 84

Quanz, S. P., Meyer, M. R., Kenworthy, M. A., et al. 2010, ApJ, 722, L49

Rafikov, R. R. 2005, ApJ, 621, L69

Rebull, L. M., Padgett, D. L., McCabe, C.-E., et al. 2010, ApJS, 186, 259

Schlegel, D. J., Finkbeiner, D. P., \& Davis, M. 1998, ApJ, 500, 525

Scholz, A., Jayawardhana, R., Wood, K., et al. 2007, ApJ, 660, 1517

Shu, F. H., Najita, J. R., Shang, H., \& Li, Z. 2000, in Protostars and Planets IV, ed. V. Mannings, A. P. Boss, \& S. S. Russell (Tucson, AZ: Univ. Arizona Press), 789

Simon, M., Dutrey, A., \& Guilloteau, S. 2000, ApJ, 545, 1034

Skrutskie, M. F., Cutri, R. M., Stiening, R., et al. 2006, AJ, 131, 1163

Spiegel, D., \& Burrows, A. 2011, ApJ, in press (arXiv:1108.5172)

Stapelfeldt, K. R., Krist, J. E., Menard, F., et al. 1998, ApJ, 502, L65

Stapelfeldt, K. R., Ménard, F., Watson, A. M., et al. 2003, ApJ, 589, 410

Thalmann, C., Grady, C. A., Goto, M., et al. 2010, ApJ, 718, L87

Toomre, A. 1964, ApJ, 139, 1217

Torres, R. M., Loinard, L., Mioduszewski, A. J., \& Rodríguez, L. F. 2009, ApJ, 698,242

Tsiganis, K., Gomes, R., Morbidelli, A., \& Levison, H. F. 2005, Nature, 435, 459

Tuthill, P., Lloyd, J., Ireland, M., et al. 2006, Proc. SPIE, 6272, 62723A

Tuthill, P. G., Monnier, J. D., Danchi, W. C., Wishnow, E. H., \& Haniff, C. A. 2000, PASP, 112, 555

Wright, J. T., Fakhouri, O., Marcy, G., et al. 2011, PASP, 123, 412

Zacharias, N. 2010, AJ, 139, 2208

Zhao, M., Gies, D., Monnier, J. D., et al. 2008, ApJ, 684, L95

Zhu, Z., Nelson, R. P., Hartmann, L., Espaillat, C., \& Calvet, N. 2011, ApJ, 729, 47 